\title{
Modular Arithmetic with Nodal Lines: Drumhead Surface States in ZrSiTe
}

\author{
Lukas Muechlerø, ${ }^{1, *}$ Andreas Topp, ${ }^{2}$ Raquel Queiroz, ${ }^{3}$ Maxim Krivenkov, ${ }^{4}$ Andrei Varykhalov, ${ }^{4}$ \\ Jennifer Cano ${ }^{1,}, 5$ Christian R. Ast, ${ }^{2}$ and Leslie M. Schoop ${ }^{6, \dagger}$ \\ ${ }^{1}$ Center for Computational Quantum Physics, The Flatiron Institute, New York, New York, 10010, USA \\ ${ }^{2}$ Max-Planck-Institut für Festkörperforschung, Heisenbergstrasse 1, D-70569 Stuttgart, Germany \\ ${ }^{3}$ Department of Condensed Matter Physics, Weizmann Institute of Science, Rehovot 76100, Israel \\ ${ }^{4}$ Helmholtz-Zentrum Berlin für Materialien und Energie, Elektronenspeicherring BESSY II, \\ Albert-Einstein-Straße 15, 12489 Berlin, Germany \\ ${ }^{5}$ Department of Physics and Astronomy, Stony Brook University, Stony Brook, New York 11974, USA \\ ${ }^{6}$ Department of Chemistry, Princeton University, Princeton, New Jersey 08544, USA
}

(Received 14 August 2019; revised manuscript received 18 November 2019; accepted 16 December 2019; published 6 February 2020)

\begin{abstract}
We study the electronic structure of the nodal line semimetal ZrSiTe both experimentally and theoretically. We find two different surface states in $\mathrm{ZrSiTe}$ - topological drumhead surface states and trivial floating band surface states, which can be easily distinguished in ARPES experiments. Using the spectra of Wilson loops, we show that a nontrivial Berry phase that exists in a confined region within the Brillouin zone gives rise to the topological drumhead-type surface states. The $\mathbb{Z}_{2}$ structure of the Berry phase induces a $\mathbb{Z}_{2}$ "modular arithmetic" of the surface states, allowing surface states derived from different nodal lines to hybridize and gap out, which can be probed by a set of Wilson loops. Our findings are confirmed by ab initio calculations and angle-resolved photoemission experiments, which are in excellent agreement with each other and the topological analysis. This work is the first complete characterization of topological surface states in the family of square-net-based nodal line semimetals, and thus it fundamentally increases the understanding of the topological nature of this growing class of topological semimetals.
\end{abstract}

DOI: $10.1103 /$ PhysRevX.10.011026

\section{INTRODUCTION}

Band inversions in three-dimensional (3D) materials can lead to a variety of topological semimetals that can be distinguished by the dimensionality and connectivity of the band touching points [1-5]. For example, Weyl semimetals are characterized by isolated points in the Brillouin zone (BZ) at which two bands cross; such crossings are protected only by translation symmetry. Protected crossings of a larger number of bands at isolated points within the BZ require the presence of additional spatial symmetries; e.g., Dirac semimetals with fourfold degenerate crossing points require the presence of rotation symmetries [6,7]. In materials with nonsymmorphic symmetries or in certain magnetic space groups, band crossings of up to eight bands can be found at high-symmetry points, which is the

\footnotetext{
*Imuechler@flatironinstitute.org

${ }^{\dagger} 1$ schoop@princeton.edu
}

Published by the American Physical Society under the terms of the Creative Commons Attribution 4.0 International license. Further distribution of this work must maintain attribution to the author(s) and the published article's title, journal citation, and DOI.
Subject Areas: Condensed Matter Physics, Topological Insulators

theoretical maximum [8-11]. In the presence of mirror symmetries, band inversions can lead to the existence of nodal lines, i.e., one-dimensional (1D) lines or loops of either twofold or fourfold degenerate band touching points in the BZ [12-15]. If multiple nodal lines are present in one material, the band structure can be further characterized by their connectivity or linking structure; e.g., it is possible for nodal lines to form knotted nodal structures that are characterized by knot invariants [16-18].

These band crossings often strongly influence the electronic properties of such topological semimetals [19]. For example, the presence of Weyl nodes in the band structure leads to the existence of Fermi arcs [20-23] on the surface of the material or to a measurable transport signature in the longitudinal magnetoresistance, the socalled chiral anomaly [24-28]. In addition, optical properties of topological semimetals are directly related to the topological invariants protecting the band crossings, such as a quantized circular photogalvanic effect $[29,30]$.

In recent years, topological nodal line semimetals have become an active field of research [5,31]. Nodal lines are characterized by local topological invariants [15], which guarantee the presence of topologically protected drumhead (DH) surface states that can be measured in 
angle-resolved photoemission spectroscopy (ARPES) experiments [32-34]. In addition, nodal lines can serve as important sources of Berry curvature or spin-Berry curvature, which contribute to observables that can be probed in transport experiments, such as the anomalous-Hall effect or the spin-Hall effect [35]. For example, it has recently been shown that the giant anomalous Hall effects in magnetic Weyl semimetals such as $\mathrm{Co}_{2} \mathrm{MnGa}$ and $\mathrm{Co}_{3} \mathrm{Sn}_{2} \mathrm{~S}_{2}$ derive from the Berry curvature around nodal lines [36,37]. Similarly, large spin-Hall effects, based on the presence of nodal lines, have been predicted in nonmagnetic compounds such as $\mathrm{RuO}_{2}$, TaAs, or $\mathrm{W}_{3} \mathrm{Ta}$ [38-40]. Furthermore, novel transport properties, such as unconventional mass enhancement or electron-hole tunneling, were discovered in nodal line semimetals $[41,42]$.

It has recently been established that the presence of nodal lines in the band structure is related to certain structural motives of the crystal structure [5,43]. For example, the family of $M X Z(M=\mathrm{Zr}$, Hf; $X=\mathrm{Si}, \mathrm{Ge} ; Z=\mathrm{S}, \mathrm{Se}, \mathrm{Te})$ materials exhibits multiple nodal lines and nonsymmorphic degeneracies right at the Fermi level due to the presence of a two-dimensional square net of $X$ atoms in its crystal structure $[44,45]$. While much attention has been directed towards the bulk properties of this class of materials, the topological drumhead surface states that are expected to derive from the presence of the nodal lines in the band structure have not been discussed in detail so far [46,47], and a general theoretical understanding is lacking. In this paper, we analyze the nodal line structure of $\mathrm{ZrSiTe}$ as a representative member of this class of materials and demonstrate the existence of topologically required surface states both theoretically and experimentally. We show that the surface states exhibit a $\mathbb{Z}_{2}$ modular arithmetic according to their $\mathbb{Z}_{2}$ quantized Berry phase, which can be probed by a set of bulk Wilson loops. ARPES data experimentally confirm the existence of these surface states in the areas of the nontrivial Berry phase.

The paper is organized as follows: In the first section, we review the electronic structure of $\mathrm{ZrSiTe}$ and compare it to its close relative $\mathrm{ZrSiS}$ with a particular emphasis on the nodal line structure. We then discuss the topological properties of $\mathrm{ZrSiTe}$ based on a Wilson loop analysis and discuss the implications for the surface states of the (001) surface. In the second section, we discuss ARPES measurements on the (001) surface of ZrSiTe and compare it with the theoretical predictions.

\section{METHODS}

\section{A. Theoretical}

The DFT calculations were performed using the VASP package [48] with the standard pseudopotentials for $\mathrm{Zr}, \mathrm{Si}$, and Te. The experimental geometries were taken from the ICSD. For the self-consistent calculations, the reducible BZ was sampled by a $7 \times 7 \times 5 k$ mesh. A Wannier interpolation using 82 bands was performed by projecting onto an atomic-orbital basis centered at the atomic positions, consisting of $\mathrm{Zr} 5 s, 6 s, 5 p, 4 d, 5 d, \quad \mathrm{Si} 3 s, 4 s$, $3 p, 4 p, 3 d$ as well as Te $5 s, 6 s, 5 p, 6 p, 5 d$ orbitals. The nodal lines and Wilson loops were calculated with an inhouse code and the WannierTools [49] package.

\section{B. Experimental}

The synthesis and characterization of $\mathrm{ZrSiTe}$ single crystals was published elsewhere [45]. ARPES experiments were performed on in situ cleaved crystals in ultrahigh vacuum (low $10^{-10} \mathrm{mbar}$ ). The spectra were recorded at $50 \mathrm{~K}$ with the $1^{2}$ ARPES experiment installed at the UE112-PGM2a beam line at the BESSY-II synchrotron.

\section{ELECTRONIC STRUCTURE}

\section{A. Nodal lines}

Just like its close relative $\mathrm{ZrSiS}$, ZrSiTe crystallizes in the nonsymmorphic space group $P 4 / \mathrm{nmm}$ (SG 129) [Fig. 1(a)]. Symmetries are key towards the understanding of the electronic structure of materials, as they protect the crossings of bands in the Brillouin zone (BZ) in highsymmetry planes, lines, or points. In the case of $\mathrm{ZrSiS}$ and $\mathrm{ZrSiTe}$, the following symmetries are of particular importance: the glide mirror $\bar{M}_{z}=\left\{M_{z} \mid \frac{1}{2} \frac{1}{2} 0\right\}$, the mirror $M_{x y}$, spatial inversion $\{I \mid 000\}$, and the two screw symmetries $\bar{C}_{2 x}=\left\{C_{2 x} \mid \frac{1}{2} 00\right\}$ and $\bar{C}_{2 y}=\left\{C_{2 y} \mid 0 \frac{1}{2} 0\right\}$. In combination with time-reversal symmetry $\mathcal{T}$, these symmetries lead to nonsymmorphically enforced degeneracies at the BZ boundary and the occurrence of multiple nodal lines. We start our analysis without accounting for spin-orbit coupling (SOC); the presence of SOC will be discussed in detail later. The combination of a screw axis with time-reversal symmetry $\mathcal{T}$ enforces double degenerate states (ignoring spin) in the M-X-A-R plane as shown in Fig. 1(b). Inside the $\mathrm{BZ}, \bar{M}_{z}$ can protect band crossings in the $k_{z}=0, \pi$ plane, while $M_{x y}$ can protect band crossings in the plane given by $k_{x}=k_{y}$. The combinations of the screw symmetries with inversion, $\bar{C}_{2 x} I$ and $\bar{C}_{2 y} I$, allow for crossings
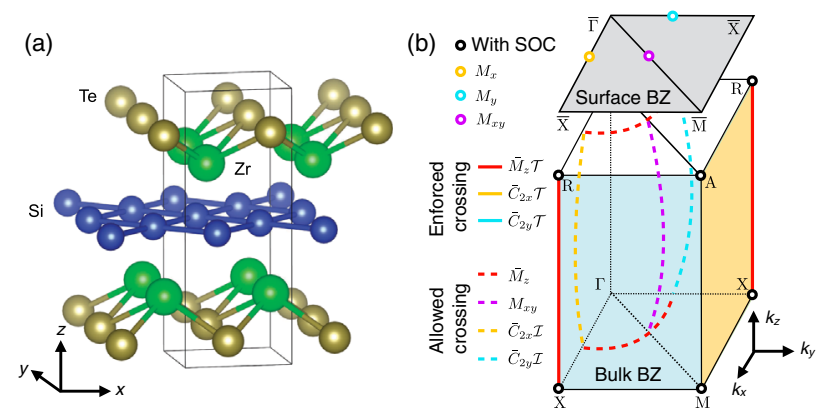

FIG. 1. (a) Crystal structure of $\mathrm{ZrSiTe}$. Si atoms are displayed in blue, $\mathrm{Zr}$ in green, and $\mathrm{Te}$ in brown. (b) Brillouin zone and crossings enforced or allowed by the space-group symmetries of $P 4 / \mathrm{nmm}$. 


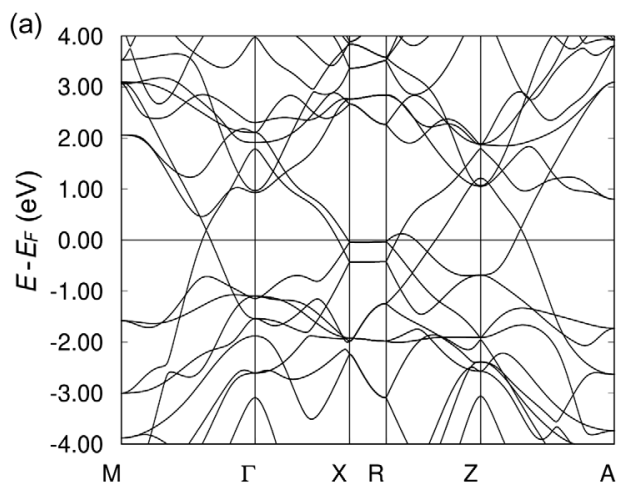

(d)

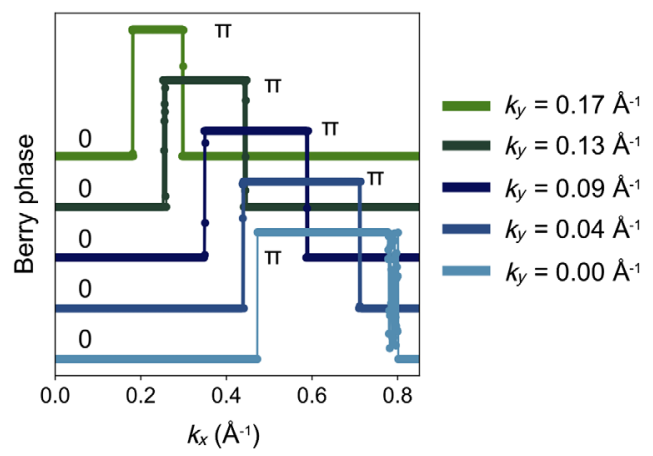

(b)

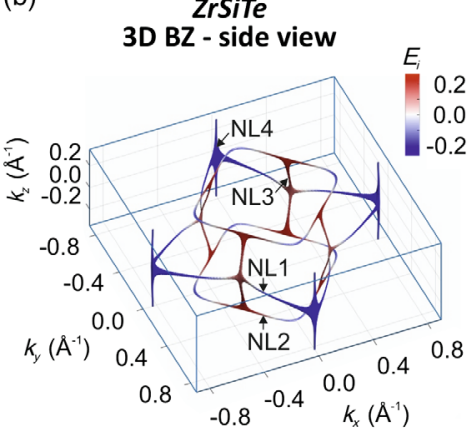

2D BZ - surface projection

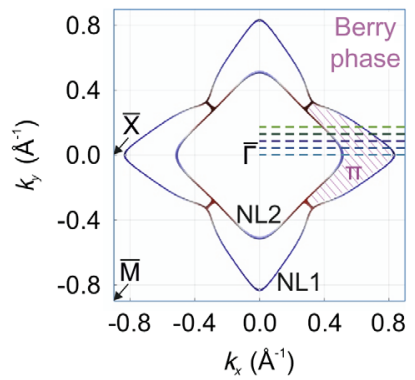

(c)
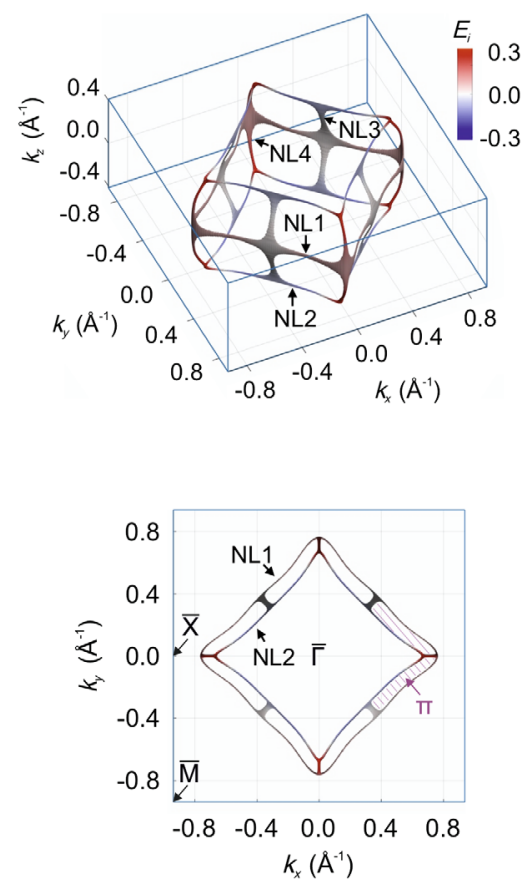

FIG. 2. (a) Bulk band structure of ZrSiTe without SOC. (b) ZrSiTe nodal line connectivity in the 3D BZ (top) and (001) surface projection (bottom). The area of the calculated $\pi$ Berry phase is indicated in purple in one quadrant. (c) Nodal line connectivity of ZrSiS analogous to panel (b). (d) Berry phase calculated from Wilson loops along $k_{z}$ as a function of $k_{x}$ for fixed $k_{y}$. A Berry phase of $\pi$ implies the presence of topological drumhead surface states at the (001) surface. The constant $k_{y}$ cuts correspond to the dashed lines in panel (b).

in the $k_{y}=0$ and $k_{x}=0$ planes, respectively. Because of these many high-symmetry planes, the band structure of $\mathrm{ZrSiTe}$ is complex with a plethora of band crossings [Fig. 2(a)]. For this reason, we only focus on crossings close to the Fermi level in the following discussion. In the vicinity of the Fermi level, two nodal lines protected by $\bar{M}_{z}$ can be found in the $k_{z}=0$ (NL1) and $k_{z}=\pi$ planes (NL2), respectively. The dispersions of NL1 and NL2 are noticeably different from each other [Fig. 2(b)], indicating that the electronic structure is of 3D character, despite ZrSiTe's layered crystal structure and the fact that bulk crystals can be easily exfoliated [50]. The two nodal lines are connected by an additional nodal line (NL3) in the perpendicular direction, which is protected by $M_{x y}$. Furthermore, a nodal line (NL4), which is protected by $\bar{C}_{2 x}$, exists along the $k_{z}$ direction that connects to the nodal line in the $k_{z}=0$ plane but does not terminate at the nodal line in the $k_{z}=\pi$ plane. The resulting cagelike nodal structure is displayed in Fig. 2(b). In contrast, in $\mathrm{ZrSiS}$, whose nodal line structure is reproduced in Fig. 2(c), the nodal lines that lie in the $k_{z}=0$ and $k_{z}=\pi$ planes (NL1 and NL2) are connected by two additional nodal lines-one protected by $M_{x y}$ and one by $\bar{C}_{2 x} I$. In summary, in ZrSiS, both NL3 and NL4 connect NL1 and NL2, while in ZrSiTe, only NL3 connects NL1 and NL2. NL1 and NL2, which have similar shapes in $\mathrm{ZrSiS}$, differ significantly in $\mathrm{ZrSiTe}$. This difference is clearly visible in the (001) surface projection of the nodal lines, as shown in the lower part of Fig. 2(b) and 2(c), and has important consequences for the presence of topological drumhead surface states.

\section{B. Wilson loops and Berry phase}

Topological surface states are expected to appear in areas of the surface BZ in which the Berry phase $\gamma$ equals $\pi$. The Berry phase can be computed by a Wilson loop directed in parallel to the surface normal vector. The Wilson loop is defined as the path-ordered exponential of the non-Abelian Berry connection $\mathbf{A}(\boldsymbol{k})_{i j}=\left\langle u_{i, \boldsymbol{k}}\left|\nabla_{\boldsymbol{k}}\right| u_{j, \boldsymbol{k}}\right\rangle$ [51-53]:

$$
\mathcal{W}(\ell)=\overline{\exp }\left[-\int_{\ell} d \boldsymbol{k} \cdot \boldsymbol{A}(\boldsymbol{k})\right],
$$

where $\left|u_{j, k}\right\rangle$ is an occupied eigenstate of the Hamiltonian and $\ell$ is a path in the $\mathrm{BZ}$ with a finite gap between the highest occupied state and the lowest unoccupied one. The Berry phase $\gamma \in[0,2 \pi)$ is determined by the Abelian part $(\operatorname{Tr} \mathbf{A})$ of the Berry connection and can be obtained from the determinant of the Wilson loop via

$$
e^{i \gamma}:=\operatorname{det}[\mathcal{W}(\ell)]=\exp \left[-\int_{\ell} d \boldsymbol{k} \cdot \operatorname{Tr} \mathbf{A}(\boldsymbol{k})\right] .
$$

It is straightforward to show that quantization of the Berry phase to either 0 or $\pi$ occurs if the path of the Wilson loop $(\ell)$ is reversed by a unitary symmetry $\boldsymbol{g}$ 
of the Hamiltonian, i.e., $g: \ell \mapsto-\ell$, as we now derive. Under this symmetry, the Wilson loop transforms as

$$
\boldsymbol{g} \mathcal{W}(\ell) \boldsymbol{g}^{-1}=\mathcal{W}(\boldsymbol{g} \ell)=\mathcal{W}(-\ell) .
$$

Making use of the fact that $\mathcal{W}(-\ell)=\mathcal{W}^{-1}(\ell)$, we arrive at

$$
\begin{aligned}
\operatorname{det}\left[\boldsymbol{g} \mathcal{W}(\ell) \boldsymbol{g}^{-1}\right] & =\operatorname{det}[\mathcal{W}(-\ell)] \\
\Rightarrow \operatorname{det}[\mathcal{W}(\ell)] & =\operatorname{det}\left[\mathcal{W}(\ell)^{-1}\right] \\
\Rightarrow \operatorname{det}[\mathcal{W}(\ell)]^{2} & =1,
\end{aligned}
$$

which implies that $\gamma=0$ or $\gamma=\pi$.

We are interested in the topological surface states of the (001) surface of $\mathrm{ZrSiTe}$, which we analyze by Wilson loops along $k_{z}$ starting from $k_{z}=-\pi$ and ending at $k_{z}=\pi$, while $k_{x}$ and $k_{y}$ remain constant. In this case, the Berry phase becomes a function of the base points $\left(k_{x}, k_{y}\right)$, i.e., $\gamma \equiv \gamma\left(k_{x}, k_{y}\right)$, while $\bar{M}_{z}$ ensures the quantization of the Berry phase to either 0 or $\pi$. In areas of the surface BZ, in which the surface projections of the nodal lines NL1 and NL2 overlap, the total Berry phase is expected to be $\gamma=2 \pi$ since each nodal line contributes $\pi$ to the Abelian part of the Wilson loop along $k_{z}$. However, since the Berry phase is defined modulo $2 \pi$, no topological surface states are expected in regions where the nodal lines project on top of each other. On the other hand, in regions of the surface where only one nodal line projects, the Berry phase will be quantized to the nontrivial value of $\pi$, and drumhead surface states are expected. Therefore, the drumhead states exhibit a $\mathbb{Z}_{2}$ modular arithmetic that follows from their topological classification through Wilson loops. Because of the larger momentum separation in the surface projection between NL1 and NL2 in ZrSiTe compared to ZrSiS, the area in which drumhead states can be observed is significantly larger for ZrSiTe as shown in Fig. 2(a) and 2(b). Figure 2(d) shows the Berry phase calculated as a function of $k_{x}$ for a set of fixed values of $k_{y}$ for all occupied bands. For small values of $k_{x}$, as long as the base point is inside the overlap region of the nodal lines NL1 and NL2, the calculated Berry phase is equal to 0. As shown in Fig. 2(d), the Berry phase adopts a value of $\pi$ in areas only one nodal line projects to, while it is zero elsewhere. The Berry phase abruptly changes between 0 and $\pi$ upon crossing a nodal line boundary. The total area in which the Berry phase equals $\pi$ is highlighted by dashed violet lines in panel (b).

So far, we have only discussed the Abelian part of the Wilson loop and its relation to the surface states. The nonAbelian part, i.e., the eigenvalues of the Wilson loop, allow us to make more detailed statements of the topological structure, in particular, the interplay between space-group symmetries, the nodal lines, and their surface states $[52,53]$. The unimodular eigenvalues $e^{i \phi_{i}}$ of the Wilson loop, whose phases $\phi \equiv \phi_{i}\left(k_{x}, k_{y}\right)$ depend on the base point of the Wilson loop, are called Wannier charge centers (WCCs) or
non-Abelian Berry phases. For a Wilson loop along the $k_{z}$ direction, $\phi_{i}\left(k_{x}, k_{y}\right)$ is related to the charge density of a Wannier function $w_{i}$ that is maximally localized in the $z$ direction. The WCC can thus be interpreted as a pointlike charge density defined within a single unit cell for a fixed base point $\left(k_{x}, k_{y}\right)$ [54,55], effectively mapping each base point to a one-dimensional problem analogous to the $\mathrm{Su}$ Schrieffer-Heeger model [56]. The sum of the WCCs is equal to the Berry phase, i.e.,

$$
\gamma=\sum_{i} \phi_{i}
$$

and is related to the electric polarization in the unit cell via $P=(e / 2 \pi) \gamma \bmod e$, where $e$ is the electric quantum of charge [57].

The positions of the WCCs are constrained by spacegroup symmetries [52,53]. In the case of $\mathrm{ZrSiTe}$, the nonsymmorphic mirror $\bar{M}_{z}$ constrains the positions of the WCCs to three locations [Fig. 3(a)]: (i) two positions with multiplicity one, located either at the location of the mirror plane at the origin (position 1a), corresponding to $\phi\left(k_{x}, k_{y}\right)=0$, or the unit-cell boundary (position $1 b$ ), (a)

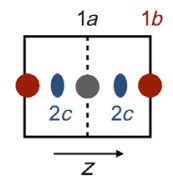

(b)

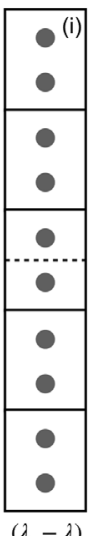

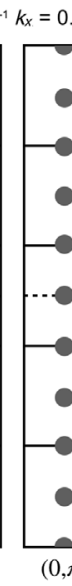

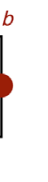

$k_{x}=0.1 \AA^{-1}$

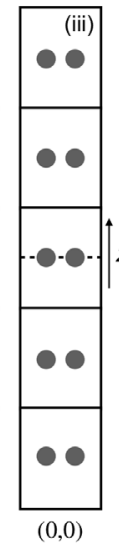

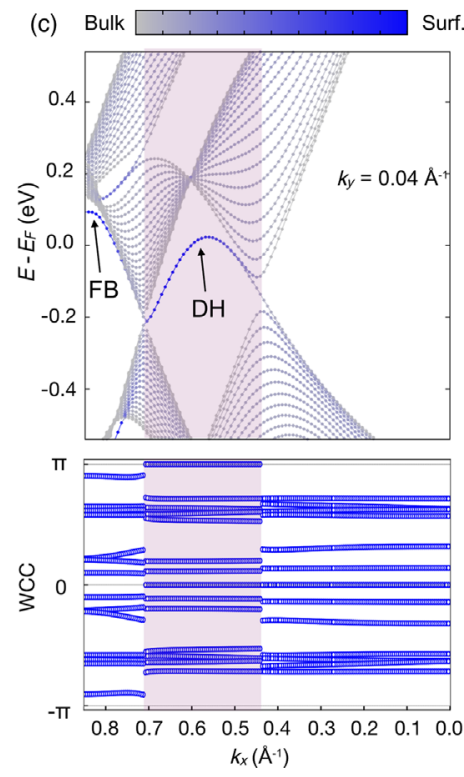

FIG. 3. (a) WCC positions constrained by $\bar{M}_{z}$ in a single unit cell. (b) Three different $\bar{M}_{z}$ symmetric slabs with different configurations of WCCs corresponding to the three different configurations found in $\mathrm{ZrSiTe}$ [compare to panel (c)]: (i) WCC located at position $2 c$, (ii) one WCC located at position $1 a$ and one at $1 b$, (iii) two WCCs located at position $1 a$. Only two WCCs are shown for the sake of clarity. (c) Surface band structure calculated from a Wannier interpolation and WCCs calculated along the same $k$ path. Bulk states are depicted in gray, while surface states are colored blue. DH surface states in regions with a $\pi$-Berry phase (shaded region) are of topological origin, while the other surface states $(\mathrm{FB})$ close to $E_{F}$ derive from the local breaking of the nonsymmorphic symmetry $\bar{M}_{z}$ at the surface [58]. 
corresponding to $\phi\left(k_{x}, k_{y}\right)=\pi$; (ii) one position with multiplicity two (position 2c), corresponding to two WCCs $\phi_{1}\left(k_{x}, k_{y}\right), \phi_{2}\left(k_{x}, k_{y}\right)$ located between positions $1 a$ and $1 b$, i.e., $\phi_{1}\left(k_{x}, k_{y}\right)=-\phi_{2}\left(k_{x}, k_{y}\right)$. An odd number of WCCs located at position $1 a$ is topologically inequivalent to the same number of WCCs located at position $1 b$ since a single WCC cannot be moved from $1 a$ to $1 b$ without breaking the mirror symmetry or going through a gap closing point.

The location of the WCC constrains the surface-state spectrum. A surface can be modeled by creating a mirror symmetric slab of unit cells along the $z$ direction [Fig. 3(b)]. Topological surface states occur if the surface termination cuts through an odd number of WCCs; in our case, this corresponds to an odd number of WCCs located at position $1 b$ and thus to a bulk Berry phase $\gamma=\pi$. The WCCs are completely determined by the $\bar{M}_{z}$ eigenvalues of the occupied states at $k_{z}=0$ and $k_{z}=\pi$ via an exact mapping that determines the eigenvalues uniquely (see the Appendix) [52].

To exemplify this mapping and to validate our topological analysis, we show the band structure of the (001) surface and the corresponding WCCs as a function of $k_{x}$ for fixed $k_{y}=0.04 \AA^{-1}$ in Fig. 3(c). The total number of occupied bands at each $k$ point is 14 , and the corresponding symmetry eigenvalues of each occupied band are shown in Table I. Topological drumhead states are clearly visible for $k$ points that lie between the projections of the two nodal lines NL1 and NL2, which is the region where the Berry phase is quantized to $\pi$. Therefore, two WCCs are quantized to $0, \pi$, respectively; i.e., both positions $1 a$ and $1 b$ are occupied. The other WCCs come in pairs $(\lambda,-\lambda)$ located on position $2 c$ [compare Fig. 3(b)]. In the region that only contains the projection of one nodal line $\left(0.44<k_{x}<0.7\right)$, the number of positive and negative $\bar{M}_{z}$ eigenvalues of the occupied bands at $k_{z}=\pi$ is equal, while there are two more

TABLE I. Multiplicity $N_{ \pm, k_{z}}$ of the positive and negative branches of the $\bar{M}_{z}$ eigenvalues in the two mirror-invariant planes $k_{z}=0, \pi$ for three selected $k_{x}$ at constant $k_{y}=0.04 \AA^{-1}$. In addition, we list the number $N_{ \pm 1}$ of Wilson-loop eigenvalues quantized to \pm 1 and number $N_{\alpha, \alpha^{*}}$ of complex-conjugate pairs $\alpha, \alpha^{*}$ obtained by the mapping discussed in the Appendix at each $k_{x}$. At each $k$ point, 14 bands are occupied.

\begin{tabular}{lccc}
\hline \hline$k_{x} / \AA^{-1}$ & 0.1 & 0.6 & 0.8 \\
\hline$N_{+, k_{z}=0}$ & 6 & 6 & 7 \\
$N_{-, k_{z}=0}$ & 8 & 8 & 7 \\
$N_{+, k_{z}=\pi}$ & 6 & 7 & 7 \\
$N_{-, k_{z}=\pi}$ & 8 & 7 & 7 \\
$N_{+1}$ & 2 & 1 & 0 \\
$N_{-1}$ & 0 & 1 & 0 \\
$N_{\alpha, \alpha^{*}}$ & $2 \times 6$ & $2 \times 6$ & $2 \times 7$ \\
\hline \hline
\end{tabular}

positive than negative eigenvalues in the $k_{z}=0$ plane. In the areas containing the projections of NL1 and NL2 $\left(k_{x}<0.44\right)$, both the number of positive and negative $\bar{M}_{z}$ eigenvalues of the occupied bands at $k_{z}=0$ and $k_{z}=\pi$ differ by two, leading to two WCCs quantized to 0 , while the others come in pairs of $(\lambda,-\lambda)$. For $k_{x}>0.7$, the number of positive and negative $\bar{M}_{z}$ eigenvalues is equal for both $k_{z}=0$ and $k_{z}=\pi$, and the WCCs occur only in pairs $(\lambda,-\lambda)$. An additional set of surface states emerges from the bulk bands in this region, despite a vanishing Berry phase. This surfaces state has been discussed before in $\mathrm{ZrSiS}$ [58] and is called the floating band (FB) surface state. It originates from the local breaking of the nonsymmorphic symmetry $\bar{M}_{z}$ at the surface and is not of topological origin. While $\bar{M}_{z}$ is essential to define the topological invariants, the breaking of the mirror symmetry through disorder does not necessarily destroy the topological protection of the drumhead states. It has been argued by Chan et al. [59] that the drumhead states remain protected, as long as the $\bar{M}_{z}$ is preserved on average and as long as the disorder can be treated perturbatively. Numerically, we find that the drumhead states remain clearly visible in a slab model in which all atomic positions have been randomly displaced based on a uniform distribution ranging from 0 to $0.1 \AA$.

\section{Spin-orbit coupling}

It is important to point out that the nodal lines in $\mathrm{ZrSiTe}$ are not stable with respect to SOC; i.e., they gap once SOC is considered since the topological protection of the nodal lines and the drumhead states is lifted when SOC is considered. Therefore, the drumhead states could be removed in materials with strong SOC. In the limit of weak SOC, however, the drumhead states are not expected to disappear, which has to be checked by explicit calculation of the surface band structure when SOC is taken into account. As we will show below, in $\mathrm{ZrSiTe}$, SOC is weak enough so that drumhead surface states are still observable. The resulting gaps at the nodal line are of the order of $0.1 \mathrm{eV}$ in $\mathrm{ZrSiTe}$, which is small compared to the bandwidths $W \sim 4 \mathrm{eV}$ of the bands giving rise to the nodal lines. Therefore, we expect that the effects of SOC can be described perturbatively with only slight changes to the topological surface states. Our analysis shows that the drumhead states on the (001) surface are twofold degenerate (counting spin) when SOC is not considered; they split into two branches upon consideration of SOC. For weak SOC, the splitting is expected to be small (on the order of a few meV according to our DFT calculations), but it is still expected to be observable experimentally. To verify this hypothesis, we calculate the surface spectral function of $\mathrm{ZrSiTe}$ with and without SOC (Fig. 4). The projections of the bulk nodal lines NL1 and NL2 are clearly visible along the $\bar{\Gamma}-\bar{X}$ line, while the projection of the nodal line NL3 can be clearly observed along $\bar{M}-\bar{\Gamma}$. Similar to the bulk states, the drumhead states are split slightly due to 

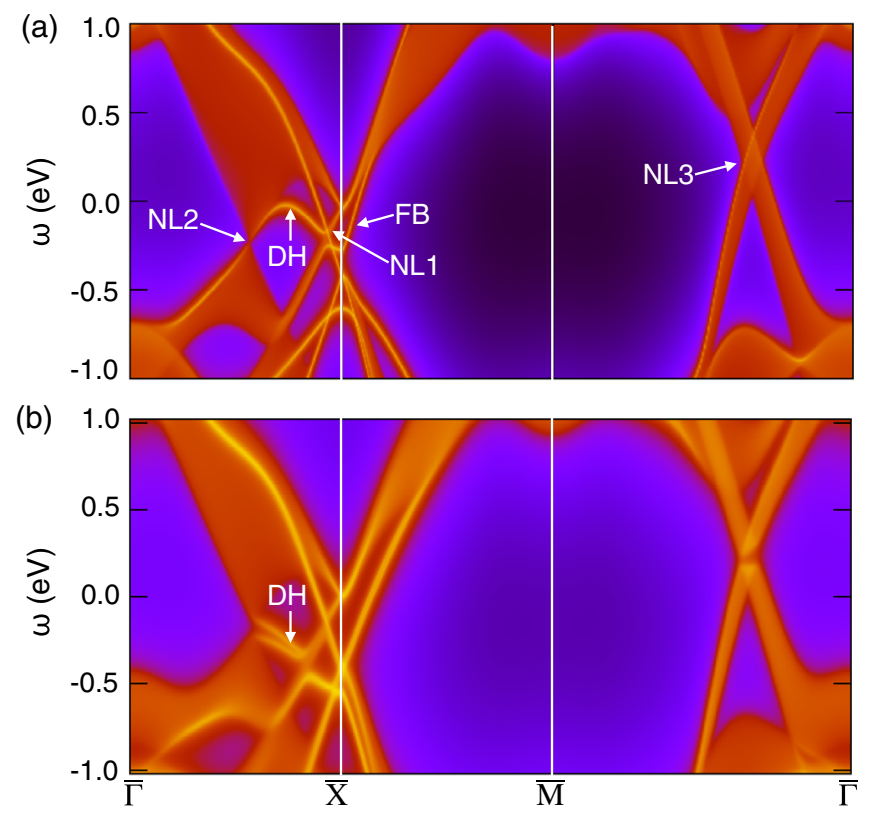

FIG. 4. Surface spectral function of $\mathrm{ZrSiTe}(001)$ along surface high-symmetry lines without SOC (a) and with SOC (b). NL1 and NL2 are the surface projections of the slightly gapped bulk nodal lines in the $k_{z}=0$ and $k_{z}=\pi$ planes. The DH emerges from the nodal line NL1 and merges into the bulk states derived from NL2. Along $\bar{X}-\bar{M}$, a FB surface state can be observed, while the projection of the bulk nodal line NL3 that connects NL1 with NL2 can be observed along $\bar{M}-\bar{\Gamma}$.

SOC, and their dispersion is shifted slightly towards lower energies. Yet, they remain clearly distinguishable features in the surface band structure, which leaves us to conclude that SOC in ZrSiTe can indeed be described as a small perturbation as far as the topological properties are concerned.

\section{ARPES MEASUREMENTS}

The topological analysis presented above can be verified with ARPES. Since ARPES experiments are only sensitive to the occupied part of the band structures, we limit our experimental analysis to the immediate vicinity of the $\bar{X}$ point, which is where the drumhead states are predicted to appear below $E_{F}$. Figure 5(a) shows a selection of constant energy cuts from $-0.3 \mathrm{eV}$ to $0 \mathrm{eV}$ close to the $\bar{X}$ point. At a photon energy of $\hbar \omega=39 \mathrm{eV}$, the surface-state contributions appear as bright features in the band structure, while contributions from the bulk nodal line are only visible with very weak intensity. It is prudent to note that a detailed photon-energy-dependent study will not allow us to unambiguously identify the surface bands around the $\mathrm{X}$ point here. As can be seen from the nondispersive bands close to the Fermi level along X-R in Fig. 2(a), the bulk bands are fairly two dimensional as well. In the following, we therefore identify the surface bands by their high intensity in combination with a comparison to bulk and slab DFT calculations. This method is in line with previous studies of surface states in this class of material that have shown that the surface states possess a large intensity for small photon energies $[46,47,60,61]$. Because of the clear difference in intensity, two crescent-shaped branches of the drumhead surface states, which are split by SOC, can be observed and are labeled in Fig. 5(b). The same two crescent-shaped drumhead surface states can also be seen in the DFTcalculated Fermi surface shown in Fig. 5(c). To gain an understanding of the total area in which the drumhead states reside and whether this area coincides with that of the nontrivial Berry phase, we trace the area of the drumhead state that lies closest to $\bar{\Gamma}$, for several initial-state energies. These regions are then superimposed to visualize the overall extent of the drumhead states [black lines shown on the left side of Fig. 5(b)]. The bare measured data are shown without the black surface-state projection on the right side of panel (b) as a comparison. Thus, the contribution of the drumhead to the Fermi surface can be evaluated directly. In Fig. 5(c), which shows the calculated Fermi surface, the left side also shows the projection of the surface states between $-0.35 \mathrm{eV}$ and $E_{\mathrm{F}}$, while the right side presents the bare calculated Fermi surface. On the left side, the bulk nodal line is superimposed as a red line on top of the surface projection. The analysis in Fig. 5(b) and 5(c) shows that, as expected, the drumhead states only exist in the region between NL1 and NL2, which is exactly the region where the Berry phase is nontrivial. In addition to the drumhead states, another high-intensity feature can be observed along the $\overline{\mathrm{X}}-\overline{\mathrm{M}}$ direction. As mentioned in Sec. III B, these states have previously been described as floating bands [58]. They also appear in the calculated Fermi surface in the same region. These two types of surface states coexist in $\mathrm{ZrSiTe}$, but they are of different electronic origin and, therefore, behave very differently in their dispersion plots. Such dispersion cuts and their theoretical counterparts are shown in Fig. 5(d) and 5(e), respectively. The dispersion plots were chosen to show constant $k_{y}$ values along and in parallel to the highsymmetry line $\bar{\Gamma}-\bar{X}$, indicated by the black lines in panels (b) and (c). In the calculated band structure, the surface bands are plotted in blue, while the bulk bands are plotted in grey. The grey shaded area represents the energy window chosen for the surface projection of the left side of panel (c), which captures the energy range occupied by the drumhead surface states up to the Fermi level. On the experimental side, the surface states can again be identified by their high intensity. In both the experimental and the theoretical data, the surface states extend down to the lowest initial-state energy along the high-symmetry line of cut $1(\simeq 0.4 \mathrm{eV})$. The drumhead states are expected to appear at slightly higher energies, while floating band states reside at lower energies, below $-0.35 \mathrm{eV}$ in the vicinity of the $\bar{X}$ point. In the measured data, the two SOC-split branches of the drumhead state can be clearly resolved by 

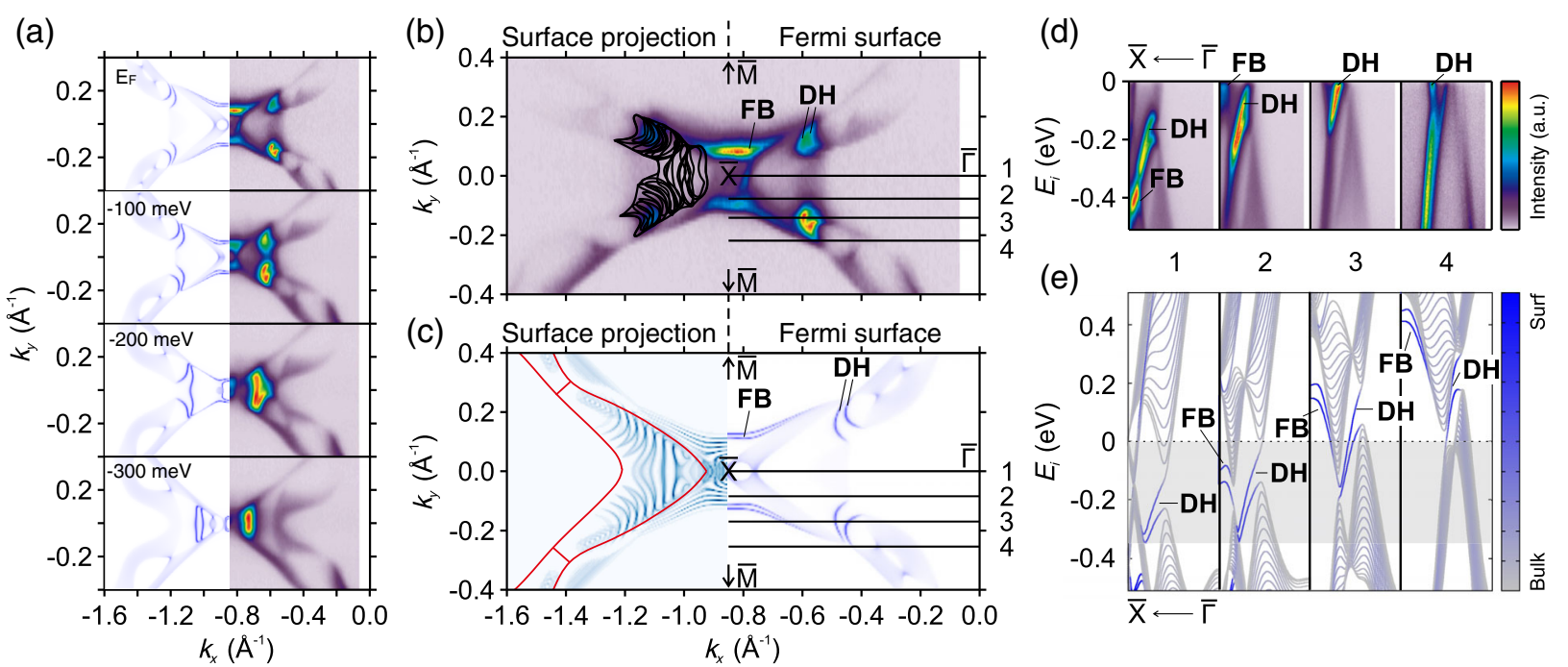

FIG. 5. ARPES data, taken at $\hbar \omega=39 \mathrm{eV}$, in comparison with DFT calculations. (a) Constant energy cuts around the $\bar{X}$ point for selected initial-state energies. (b) Magnification of the Fermi surface, showing the experimentally determined drumhead surface-state projection on the left. Two different highly intense surface states are labeled as DH and FB. (c) Slab calculations in analogy to panel (b). The nodal line is superimposed on the surface projection in red. (d,e) Dispersion cuts in experiment and DFT for constant $k_{y}$ values according to the black lines in panels (b) and (c), respectively. The FB disperses much stronger, while the DH is located inside the nodal line overlap.

connecting the bulk nodal lines, which are visible as dark shadows in the chosen color scale. Moving away from the high-symmetry line $\bar{\Gamma}-\bar{X}$ (following cuts $2-4$ ), we can observe the drumhead surface state to slowly disperse upwards in energy, while it remains between the surface projection of the bulk nodal line, in agreement with the calculations. In cut 4, the lower branch of the drumhead surface state barely remains in the picture. The floating bands, on the other hand, show a very different behavior. They exhibit a much steeper dispersion along $k_{y}$ and move above the Fermi level very rapidly; by cut 3 , they are no longer visible.

\section{CONCLUSION}

In conclusion, we have studied the electronic structure of the (001) surface of the nodal line semimetal $\mathrm{ZrSiTe}$, both theoretically and experimentally. We find that the nodal lines in the $k_{z}=0$ and $k_{z}=\pi$ planes of bulk $\mathrm{ZrSiTe}$ give rise to clearly recognizable topological drumhead surface states close to $E_{\mathrm{F}}$, which remain clearly identifiable if SOC is considered. The drumhead states exhibit a $\mathbb{Z}_{2}$ modular arithmetic that follows from the topological classification through Wilson loops. As a consequence, the drumhead states gap in regions where they overlap, leading to a ribbon of drumhead states confined by the surface projections of the bulk nodal lines due to their $\mathbb{Z}_{2}$ classification [46,47]. In addition to these topologically required surface states, we find topologically trivial floating band states close to the surface high-symmetry $\bar{X}$ point. These states derive from surface symmetry breaking and have been previously reported in the closely related compound $\mathrm{ZrSiS}$. The study of such an interplay of topologically trivial and nontrivial surface states is not limited to $\mathrm{ZrSiTe}$ and should be observable in other nodal line semimetals.

\section{ACKNOWLEDGMENTS}

We would like to than Yan Sun (CPFS) for providing the Wannier interpolation. This work was partially supported by NSF through the Princeton Center for Complex Materials, a Materials Research Science and Engineering Center, DMR-1420541. The authors gratefully acknowledge the financial support by the Max Planck Society. We thank Helmholtz Zentrum Berlin for the allocation of synchrotron radiation beamtime. This work was additionally supported by the DFG, Proposal No. SCHO1730/1-1. The Flatiron Institute is a division of the Simons Foundation.

\section{APPENDIX: MAPPING BETWEEN WILSON LOOP SPECTRUM AND SYMMETRY EIGENVALUES OF OCCUPIED BANDS}

The unimodular spectrum of a Wilson loop $\mathcal{W}(\ell)$ in conjunction with a unitary symmetry $\boldsymbol{g}$ that maps $\boldsymbol{g}: \ell \mapsto$ $-\ell$ consists of complex conjugate pairs $\left(\alpha, \alpha^{*}\right)$ and eigenvalues that are quantized to \pm 1 . The number of complex conjugate pairs $N_{\alpha, \alpha^{*}}$ and the number $N_{ \pm 1}$ of eigenvalues at \pm 1 are completely determined by the eigenvalues of the symmetry $\boldsymbol{g}$ in the space of the occupied bands.

In this Appendix, we briefly review the algorithm to determine the spectrum of the Wilson loop and refer the reader to the work of Alexandradinata et al. [51,52] and Muechler et al. [53] for a more detailed derivation in the 
case of symmorphic and nonsymmorphic symmetries, respectively.

The algorithm is applicable to symmetries $\boldsymbol{g}$ that leave the base point $\boldsymbol{k}$ of the Wilson loop invariant while reversing the path $\ell$, which is parametrized by a $k$ vector $\boldsymbol{k}_{\|}$. For each base point $\boldsymbol{k}$, the symmetry commutes with the Hamiltonian at $\boldsymbol{k}_{\|}=-\boldsymbol{k}_{\|} \bmod \boldsymbol{G}$, where $\boldsymbol{G}$ is a reciprocal lattice vector, and the eigenstates of the Hamiltonian can be labeled by the eigenvalues of $\boldsymbol{g}$. For example, in $\mathrm{ZrSiTe}$, $\boldsymbol{g} \equiv \bar{M}_{z}$, while $\boldsymbol{k}=\left(k_{x}, k_{y}\right)$ and $\boldsymbol{k}_{\|}=k_{z} \hat{\boldsymbol{k}}_{z}$; i.e., the electronic states at $\left(\boldsymbol{k}, k_{z}=0, \pm \pi\right)$ can be labeled by their $\bar{M}_{z}$ eigenvalues. The algorithm to determine the spectrum of $\mathcal{W}(\ell)$ for a Wilson loop along $\boldsymbol{k}_{z}$ is then given as follows:

(i) Determine the set $N=\left\{N_{+, 0}, N_{-, 0}, N_{+, \pi}, N_{-, \pi}\right\}$, where $N_{ \pm, k_{z}=0, \pi}$ is the number of occupied bands at $\left(\boldsymbol{k}, k_{z}=0, \pm \pi\right)$, which belongs to the positive (negative) branch of $\bar{M}_{z}$ eigenvalues $\pm \exp \left\{-i\left[\left(k_{x}+k_{y}\right) / 2\right]\right\}$.

(ii) Choose the smallest number of this set. The smallest set might be empty and/or not unique, in which case any choice between the equally small sets is valid. We label the smallest number as $N_{\xi, k_{z}}$, where $\xi$ labels the branch of $\bar{M}_{z}$ and $k_{z}=0, \pi$. It is useful to define $\bar{k}_{z}$, where $\bar{k}_{z}=0$ if $k_{z}=\pi$ or $\bar{k}_{z}=\pi$ if $k_{z}=0$.

(iii) The number $N_{-\xi}$ of $-\xi$ eigenvalues is given as $N_{-\xi}=N_{+, \bar{k}_{z}}-N_{\xi, \bar{k}_{z}}$.

(iv) The number $N_{\xi}$ of $\xi$ eigenvalues is given as $N_{\xi}=N_{-, \bar{k}_{z}}-N_{\xi, k_{z}}$.

(v) The number $N_{\alpha, \alpha^{*}}$ of eigenvalues appearing in complex conjugate pairs is given as $N_{\alpha, \alpha^{*}}=2 \times N_{\xi, k_{z}}$.

We now illustrate the algorithm with an example, using the first column of Table I, which we reproduce here for convenience:

$N=\left\{N_{+, 0}=6, N_{-, 0}=8, N_{+, \pi}=6, N_{-, \pi}=8\right\}$.

We choose the smallest integer to be $N_{\xi, k} \equiv N_{+, 0}=6$; therefore, $\xi=+1, k=0$, and $\bar{k}=\pi$. We thus arrive at $N_{-1}=N_{+, \pi}-N_{+, 0}=6-6=0$, while $N_{+1}=N_{-, \pi}-$ $N_{+, 0}=8-6=2$ and $N_{\alpha, \alpha^{*}}=2 \times N_{+, 0}=12$.

[1] B. Yan and C. Felser, Topological Materials: Weyl Semimetals, Annu. Rev. Condens. Matter Phys. 8, 337 (2017).

[2] N. P. Armitage, E. J. Mele, and A. Vishwanath, Weyl and Dirac Semimetals in Three-Dimensional Solids, Rev. Mod. Phys. 90, 015001 (2018).

[3] S.-Y. Yang, H. Yang, E. Derunova, S. S. P. Parkin, B. Yan, and M. N. Ali, Symmetry Demanded Topological NodalLine Materials, Adv. Phys. X 3, 1414631 (2018).

[4] L. M. Schoop, F. Pielnhofer, and B. V. Lotsch, Chemical Principles of Topological Semimetals, Chem. Mater. 30, 3155 (2018).
[5] S. Klemenz, S. Lei, and L. M. Schoop, Topological Semimetals in Square-Net Materials, Annu. Rev. Mater. Res. 49, 185 (2019).

[6] B.-J. Yang and N. Nagaosa, Classification of Stable ThreeDimensional Dirac Semimetals with Nontrivial Topology, Nat. Commun. 5, 4898 (2014).

[7] Q. D. Gibson, L. M. Schoop, L. Muechler, L. S. Xie, M. Hirschberger, N. P. Ong, R. Car, and R. J. Cava, ThreeDimensional Dirac Semimetals: Design Principles and Predictions of New Materials, Phys. Rev. B 91, 205128 (2015).

[8] B. Bradlyn, J. Cano, Z. Wang, M. G. Vergniory, C. Felser, R. J. Cava, and B. A. Bernevig, Beyond Dirac and Weyl Fermions: Unconventional Quasiparticles in Conventional Crystals, Science 353, aaf5037 (2016).

[9] B. J. Wieder, Y. Kim, A. M. Rappe, and C. L. Kane, Double Dirac Semimetals in Three Dimensions, Phys. Rev. Lett. 116, 186402 (2016).

[10] L. M. Schoop, A. Topp, J. Lippmann, F. Orlandi, L. Müchler, M. G. Vergniory, Y. Sun, A. W. Rost, V. Duppel, M. Krivenkov et al., Tunable Weyl and Dirac States in the Nonsymmorphic Compound CeSbTe, Sci. Adv. 4, eaar2317 (2018).

[11] J. Cano, B. Bradlyn, and M. G. Vergniory, Multifold Nodal Points in Magnetic Materials, APL Mater. 7, 101125 (2019).

[12] Y. Kim, B. J. Wieder, C. L. Kane, and A. M. Rappe, Dirac Line Nodes in Inversion-Symmetric Crystals, Phys. Rev. Lett. 115, 036806 (2015).

[13] Y. H. Chan, C. K. Chiu, M. Y. Chou, and A. P. Schnyder, $\mathrm{Ca}_{3} \mathrm{P}_{2}$ and Other Topological Semimetals with Line Nodes and Drumhead Surface States, Phys. Rev. B 93, 205132 (2016).

[14] A. Yamakage, Y. Yamakawa, Y. Tanaka, and Y. Okamoto, Line-Node Dirac Semimetal and Topological Insulating Phase in Noncentrosymmetric Pnictides $\operatorname{CaAg} X(X=\mathrm{P}$, As), J. Phys. Soc. Jpn 85, 013708 (2015).

[15] C.-K. Chiu and A. P. Schnyder, Classification of ReflectionSymmetry-Protected Topological Semimetals and Nodal Superconductors, Phys. Rev. B 90, 205136 (2014).

[16] T. Bzdušek, Q. S. Wu, A. Rüegg, M. Sigrist, and A. A. Soluyanov, Nodal-Chain Metals, Nature (London) 538, 75 (2016).

[17] R. Bi, Z. Yan, L. Lu, and Z. Wang, Nodal-Knot Semimetals, Phys. Rev. B 96, 201305(R) (2017).

[18] M. Ezawa, Topological Semimetals Carrying Arbitrary Hopf Numbers: Fermi Surface Topologies of a Hopf Link, Solomon's Knot, Trefoil Knot, and Other Linked Nodal Varieties, Phys. Rev. B 96, 041202(R) (2017).

[19] J. Hu, S.-Y. Xu, N. Ni, and Z. Mao, Transport of Topological Semimetals, Annu. Rev. Mater. Res. 49, 207 (2019).

[20] B. Q. Lv, H. M. Weng, B. B. Fu, X. P. Wang, H. Miao, J. Ma, P. Richard, X. C. Huang, L. X. Zhao, G. F. Chen, Z. Fang, X. Dai, T. Qian, and H. Ding, Experimental discovery of Weyl semimetal TaAs, Phys. Rev. X 5, 031013 (2015).

[21] L. X. Yang, Z. K. Liu, Y. Sun, H. Peng, H. F. Yang, T. Zhang, B. Zhou, Y. Zhang, Y. F. Guo, M. Rahn et al., Weyl Semimetal Phase in the nNon-centrosymmetric Compound TaAs, Nat. Phys. 11, 728 (2015). 
[22] S.-M. Huang, S.-Y. Xu, I. Belopolski, C.-C. Lee, G. Chang, B. Wang, N. Alidoust, G. Bian, M. Neupane, C. Zhang et al., A Weyl Fermion Semimetal with Surface Fermi Arcs in the Transition Metal Monopnictide TaAs Class, Nat. Commun. 6, 7373 (2015).

[23] S.-Y. Xu, I. Belopolski, N. Alidoust, M. Neupane, G. Bian, C. Zhang, R. Sankar, G. Chang, Z. Yuan, C.-C. Lee et al., Discovery of a Weyl Fermion Semimetal and Topological Fermi Arcs, Science 349, 613 (2015).

[24] X. Huang, L. Zhao, Y. Long, P. Wang, D. Chen, Z. Yang, H. Liang, M. Xue, H. Weng, Z. Fang et al., Observation of the Chiral-Anomaly-Induced Negative Magnetoresistance in 3D Weyl Semimetal TaAs, Phys. Rev. X 5, 031023 (2015).

[25] F. Arnold, C. Shekhar, S.-C. Wu, Y. Sun, R. D. Dos Reis, N. Kumar, M. Naumann, M. O. Ajeesh, M. Schmidt, A. G. Grushin et al., Negative Magnetoresistance without WellDefined Chirality in the Weyl Semimetal TaP, Nat. Commun. 7, 11615 (2016).

[26] C.-L. Zhang, S.-Y. Xu, I. Belopolski, Z. Yuan, Z. Lin, B. Tong, G. Bian, N. Alidoust, C.-C. Lee, S.-M. Huang et al., Signatures of the Adler-Bell-Jackiw Chiral Anomaly in a Weyl Fermion Semimetal, Nat. Commun. 7, 10735 (2016).

[27] M. Hirschberger, S. Kushwaha, Z. Wang, Q. Gibson, S. Liang, C. A. Belvin, B. A. Bernevig, R. J. Cava, and N. P. Ong, The Chiral Anomaly and Thermopower of Weyl Fermions in the Half-Heusler GdPtBi, Nat. Mater. 15, 1161 (2016).

[28] S. Liang, J. Lin, S. Kushwaha, J. Xing, N. Ni, R. J. Cava, and N. P. Ong, Experimental Tests of the Chiral Anomaly Magnetoresistance in the Dirac-Weyl Semimetals $\mathrm{Na}_{3} \mathrm{Bi}$ and GdPtBi, Phys. Rev. X 8, 031002 (2018).

[29] F. de Juan, A. G. Grushin, T. Morimoto, and J. E. Moore, Quantized Circular Photogalvanic Effect in Weyl Semimetals, Nat. Commun. 8, 15995 (2017).

[30] Q. Ma, S.-Y. Xu, C.-K. Chan, C.-L. Zhang, G. Chang, Y. Lin, W. Xie, T. Palacios, H. Lin, S. Jia et al., Direct Optical Detection of Weyl Fermion Chirality in a Topological Semimetal, Nat. Phys. 13, 842 (2017).

[31] R. Yu, Z. Fang, X. Dai, and H. Weng, Topological Nodal Line Semimetals Predicted from First-Principles Calculations, Front. Phys. 12, 127202 (2017).

[32] G. Bian, T.-R. Chang, R. Sankar, S.-Y. Xu, H. Zheng, T. Neupert, C.-K. Chiu, S.-M. Huang, G. Chang, I. Belopolski et al., Topological Nodal-Line Fermions in Spin-Orbit Metal PbTaSe ${ }_{2}$, Nat. Commun. 7, 10556 (2016).

[33] I. Belopolski, D. S. Sanchez, G. Chang, K. Manna, B. Ernst, S.-Y. Xu, S. S. Zhang, H. Zheng, J. Yin, B. Singh et al., A Three-Dimensional Magnetic Topological Phase, Science 365, 1278 (2019).

[34] Z. Liu, R. Lou, P. Guo, Q. Wang, S. Sun, C. Li, S. Thirupathaiah, A. Fedorov, D. Shen, K. Liu, H. Lei, and S. Wang, Experimental Observation of Dirac Nodal Links in Centrosymmetric Semimetal $\mathrm{TiB}_{2}$, Phys. Rev. X 8, 031044 (2018).

[35] K. Manna, Y. Sun, L. Muechler, J. Kübler, and C. Felser, Heusler, Weyl and Berry, Nat. Rev. Mater. 3, 244 (2018).

[36] K. Manna, L. Muechler, T.-H. Kao, R. Stinshoff, Y. Zhang, J. Gooth, N. Kumar, G. Kreiner, K. Koepernik, R. Car, J. Kübler, G. H. Fecher, C. Shekhar, Y. Sun, and C. Felser, From Colossal to Zero: Controlling the Anomalous Hall
Effect in Magnetic Heusler Compounds via Berry Curvature Design, Phys. Rev. X 8, 041045 (2018).

[37] E. Liu et al., Giant Anomalous Hall Effect in a Ferromagnetic Kagome-Lattice Semimetal, Nat. Phys. 14, 1125 (2018).

[38] Y. Sun, Y. Zhang, C. Felser, and B. Yan, Strong Intrinsic Spin Hall Effect in the TaAs Family of Weyl Semimetals, Phys. Rev. Lett. 117, 146403 (2016).

[39] Y. Sun, Y. Zhang, C.-X. Liu, C. Felser, and B. Yan, Dirac Nodal Lines and Induced Spin Hall Effect in Metallic Rutile Oxides, Phys. Rev. B 95, 235104 (2017).

[40] E. Derunova, Y. Sun, C. Felser, S. S. P. Parkin, B. Yan, and M. N. Ali, Giant Intrinsic Spin Hall Effect in $\mathrm{W}_{3} \mathrm{Ta}$ and Other A15 Superconductors, Sci. Adv. 5, eaav8575 (2019).

[41] S. Pezzini, M. R. Van Delft, L. M. Schoop, B. V. Lotsch, A. Carrington, M. I. Katsnelson, N. E. Hussey, and S. Wiedmann, Unconventional Mass Enhancement around the Dirac Nodal Loop in ZrSiS, Nat. Phys. 14, 178 (2018).

[42] M. R. Van Delft, S. Pezzini, T. Khouri, C. S. A. Müller, M. Breitkreiz, L. Mareike Schoop, A. Carrington, N. E. Hussey, and S. Wiedmann, Electron-Hole Tunneling Revealed by Quantum Oscillations in the Nodal-Line Semimetal HfSiS, Phys. Rev. Lett. 121, 256602 (2018).

[43] Y.-H. Chan, C.-K. Chiu, M. Y. Chou, and A. P. Schnyder, $\mathrm{Ca}_{3} \mathrm{P}_{2}$ and Other Topological Semimetals with Line Nodes and Drumhead Surface States, Phys. Rev. B 93, 205132 (2016).

[44] L. M. Schoop, M. N. Ali, C. Straßer, A. Topp, A. Varykhalov, D. Marchenko, V. Duppel, S. S. P. Parkin, B. V. Lotsch, and C.R. Ast, Dirac Cone Protected by Non-symmorphic Symmetry and Three-Dimensional Dirac Line Node in ZrSiS, Nat. Commun. 7, 11696 (2016).

[45] A. Topp, J. M. Lippmann, A. Varykhalov, V. Duppel, B. V. Lotsch, C. R. Ast, and L. M. Schoop, Non-symmorphic Band Degeneracy at the Fermi Level in ZrSiTe, New J. Phys. 18, 125014 (2016).

[46] T. Nakamura, S. Souma, Z. Wang, K. Yamauchi, D. Takane, H. Oinuma, K. Nakayama, K. Horiba, H. Kumigashira, T. Oguchi, T. Takahashi, Y. Ando, and T. Sato, Evidence for Bulk Nodal Loops and Universality of Dirac-Node Arc Surface States in $\mathrm{ZrGe} X_{c}\left(X_{c}=\mathrm{S}, \mathrm{Se}, \mathrm{Te}\right)$, Phys. Rev. B 99, 245105 (2019).

[47] B. Fu, C. Yi, T. Zhang, M. Caputo, J. Ma, X. Gao, B. Lv, L. Kong, Y. Huang, M. Shi, S. Vladimir, C. Fang, H. Weng, Y. Shi, T. Qian, and H. Ding, Dirac Nodal Surfaces and Nodal Lines in ZrSiS, Sci. Adv. 5, eaau6459 (2019).

[48] G. Kresse and J. Furthmüller, Efficiency of Ab-Initio Total Energy Calculations for Metals and Semiconductors Using a Plane-Wave Basis Set, Comput. Mater. Sci. 6, 15 (1996).

[49] Q. S. Wu, S. N. Zhang, H.-F. Song, M. Troyer, and A. A. Soluyanov, WannierTools: An Open-Source Software Package for Novel Topological Materials, Comput. Phys. Commun. 224, 405 (2018).

[50] J. Hu, Z. Tang, J. Liu, X. Liu, Y. Zhu, D. Graf, K. Myhro, S. Tran, C. N. Lau, J. Wei et al., Evidence of Topological Nodal-Line Fermions in ZrSiSe and ZrSiTe, Phys. Rev. Lett. 117, 016602 (2016).

[51] A. Alexandradinata, Z. Wang, and B. A. Bernevig, Topological Insulators from Group Cohomology, Phys. Rev. X 6 , 021008 (2016). 
[52] A. Alexandradinata, X. Dai, and B. A. Bernevig, WilsonLoop Characterization of Inversion-Symmetric Topological Insulators, Phys. Rev. B 89, 155114 (2014).

[53] L. Muechler, A. Alexandradinata, T. Neupert, and R. Car, Topological Nonsymmorphic Metals from Band Inversion, Phys. Rev. X 6, 041069 (2016).

[54] J. Zak, Band Center-A Conserved Quantity in Solids, Phys. Rev. Lett. 48, 359 (1982).

[55] J. Zak, Berry's Phase for Energy Bands in Solids, Phys. Rev. Lett. 62, 2747 (1989).

[56] W. P. Su, J. R. Schrieffer, and A. J. Heeger, Solitons in Polyacetylene, Phys. Rev. Lett. 42, 1698 (1979).

[57] R. D. King-Smith and D. Vanderbilt, Theory of Polarization of Crystalline Solids, Phys. Rev. B 47, 1651 (1993).

[58] A. Topp, R. Queiroz, A. Grüneis, L. Müchler, A. W. Rost, A. Varykhalov, D. Marchenko, M. Krivenkov, F. Rodolakis,
J. L. McChesney, B. V. Lotsch, L. M. Schoop, and C. R. Ast, Surface Floating 2D Bands in Layered Nonsymmorphic Semimetals: ZrSiS and Related Compounds, Phys. Rev. X 7, 041073 (2017).

[59] Y.-H. Chan, C.-K. Chiu, M. Y. Chou, and A. P. Schnyder, $\mathrm{Ca}_{3} \mathrm{P}_{2}$ and Other Topological Semimetals with Line Nodes and Drumhead Surface States, Phys. Rev. B 93, 205132 (2016).

[60] C. Chen et al., Dirac Line Nodes and Effect of Spin-Orbit Coupling in the Nonsymmorphic Critical Semimetals MSiS ( $M=$ Hf, Zr), Phys. Rev. B 95, 125126 (2017).

[61] D. Takane, Z. Wang, S. Souma, K. Nakayama, C. X. Trang, T. Sato, T. Takahashi, and Y. Ando, Dirac-Node Arc in the Topological Line-Node Semimetal HfSiS, Phys. Rev. B 94, 121108(R) (2016). 